\title{
MODELLING THE FAST FLUORESCENCE RISE OF PHOTOSYNTHESIS
}

\author{
- Ellen BaAke and Johannes P. Schlöder \\ Institut für Mathematik, \\ Universität Augsburg, \\ Universitätsstr. 8, \\ D-W-8900 Augsburg, Germany \\ (E.mail:ellen@mathh4.mathematik.uni-augsburg.de)
}

\begin{abstract}
We construct an ODE model for the fast fluorescence rise of photosynthesis by combining the current reaction scheme of the PS II two-electron-gate with a quasi steady-state description of the fast processes of excitation energy transfer and primary charge separation. The model is fitted to measured induction curves with a multiple shooting algorithm, and remarkably good approximations of the data are obtained. Model refinements are discussed focusing on PS II heterogeneity, and on PS I.
\end{abstract}

1. Introduction. The fluorescence of the photosynthetic apparatus is a gift of nature to those studying photosynthesis. It emerges from the chlorophylls which absorb light and, while largely converting it into chemical energy, reemit a small fraction as fluorescence.

With the onset of photosynthesis (after a longer dark interval, for example), conspicuous changes in the chlorophyll fluorescence take place which can be measured easily and precisely. This is why these phenomena were discovered very early and have remained irreplaceable in photosynthesis research until today.

One of the most well-known induction effects is the "fast fluorescence rise" observed during the first second(s) of illumination (see Fig. 1). Although described as early as 1931 by Kautsky (1931), its prominent biphasic behaviour (to which it owes its name of "OIDP-kinetics"-origin, inflection point, dip, peak) has remained a Gordian knot until today. From numerous papers, some working hypotheses have emerged which remain ultimately contradictory: the two most favoured explanations ascribe the phenomenon to photosystem I (PS I), and to the PS II non-B-type centres, respectively (for reviews, see Briantais et al., 1986; Duysens, 1986; Govindjee and Satoh, 1986; Krause and Weis, 1991).

On the other hand, the OIDP rise is most important as an experimental tool both in basic photosynthesis research and in practical applications (for a review, see Renger and Schreiber, 1987). Many external factors (e.g. freezing stress, herbicides) give rise to characteristic changes in the fast fluorescence rise 


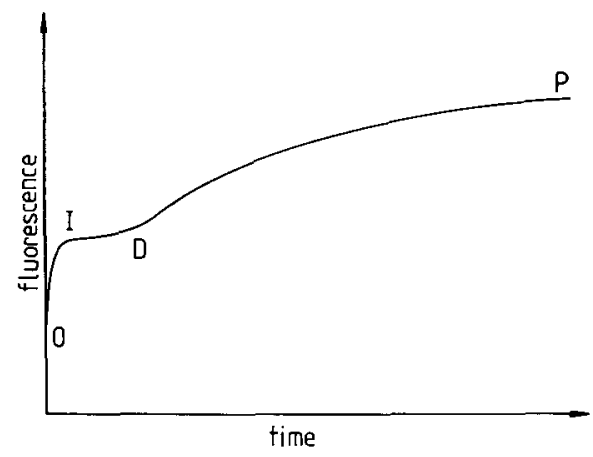

Figure 1. The fast fluorescence rise curve (schematically). $\mathrm{O}$ origin, I inflection point, D dip, P peak.

which provoke qualitative and quantitative conclusions. Such interpretations must, however, be considered with scepticism as long as the phenomenon itself is ultimately unresolved.

Some light may be thrown onto the conflicting qualitative ideas by modelling the proposed mechanisms and comparing them with the experimental data quantitatively. Previous models of fluorescence kinetics have either focused on electron transport through the two-electron-gate on the millisecond timescale (Renger and Schulze, 1985), or on the fast (nano-microsecond timescale) processes of light absorption, transfer of excited states, and charge separation (Schatz et al., 1988; Leibl et al., 1989). In this paper, whose main results are taken from Baake (1989), we modify these and bring them together in a consistent way, which yields our "core model" of fluorescence induction. We then illustrate the procedure of fitting the model to measured induction curves. Finally, the core model is extended according to the aforementioned hypotheses for the OIDP rise and again compared to the data. The results are discussed with respect to our current understanding of the light reaction and fluorescence.

2. Basic Mechanisms and the Core Model. The fluorescence commonly measured in induction experiments (wavelengths around $685 \mathrm{~nm}$ ) originates nearly exclusively from photosystem II (see Krause and Weis, 1991), so a core model for its description is naturally a model of PS II reaction kinetics. Two different time scales must be considered.

What happens at the slow (millisecond) time scale is depicted in Fig. 2 (adapted from Renger and Schulze, 1985), the reaction scheme of PS II electron transport through the so-called "two-electron-gate" (Crofts and Wraight, 1983). Charges generated at the primary acceptor $Q_{A}$ by the light-driven charge separation (see below) move on to the secondary acceptor $Q_{B}$ which, after two such steps, exchanges two electrons with a mobile pool of 


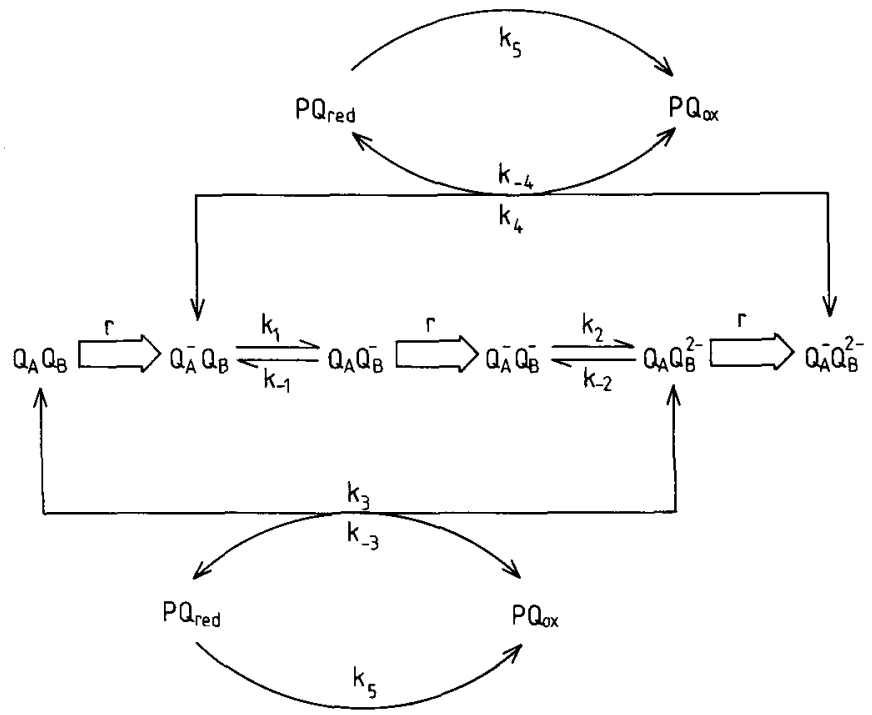

Figure 2. Scheme of the PS II two-electron-gate (adapted from Renger and Schulze, 1985 ). $Q_{A}$ : primary (stable) acceptor. $Q_{B}$ : secondary acceptor. PQ: plastoquinone.

plastoquinone (PQ) molecules. The rest of the electron transport chain, proceeding from PQ to PS I, lends itself to simplification, because the reoxidation of $\mathrm{PQ}$ is the rate-limiting step of the whole reaction sequence; we thus represent it by a simple quasi-linear reoxidation as a first approximation (PS I will be reincluded later).

The quantity $r$ characterizing the rate of the light step is not a rate constant (as are the $k$ 's) but a rate function, which will be obvious when a zoom is put on the light step. On the fast (nanosecond-microsecond) time scale, it must be resolved further into the elementary steps of light absorption, transfer of excited states, and the primary charge separation. We propose the reaction scheme shown in Fig. 3, which relies on the results of Schatz et al. (1988) and Leibl et al. (1989), and additionally includes the possibility of excitation energy transfer between neighbouring units.

The fast processes take place at the level of the so-called photosynthetic units (PSU's) which consist of a reaction centre (RC) connected to a pool of antenna chlorophyll molecules (Chl), the intermediary (unstable) electron acceptor pheophytin (Phe), and the primary stable acceptor $\mathrm{Q}_{A}$ already encountered in the slow scheme (the secondary acceptor $\mathrm{Q}_{B}$ is not considered here because its redox state is not known to have any influence on the fast processes).

Light absorption $\left(k_{L}, k_{L}\right)$ transfers the ground state $(\mathrm{Chl}-\mathrm{RC}) \mathrm{PheQ}_{A}^{(-)}$into an excited state $(\mathrm{Chl}-\mathrm{RC})^{*} \mathrm{PheQ}_{A}^{(-)}$(the excitation originally located at one particular antenna molecule equilibrates over the whole antenna within picoseconds; Schatz et al., 1988). The excited state reversibly decays $\left(\kappa_{1}, \bar{\kappa}_{1}\right.$, 


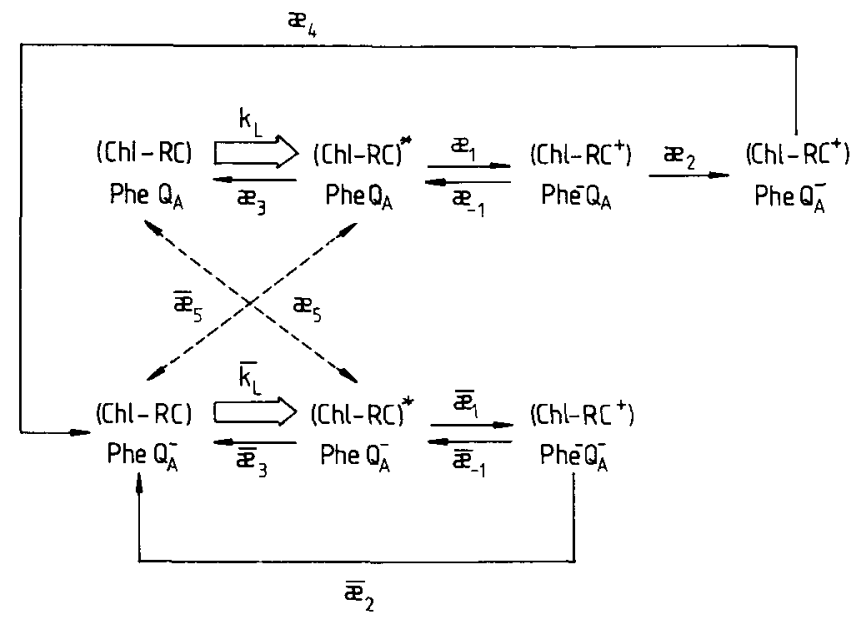

Figure 3. Scheme of fast PS II events. Chl: complex of antenna chlorophyll molecules. RC: reaction centre. Phe: pheophytin (intermediary electron acceptor). $\mathrm{Q}_{A}$ : primary (stable) acceptor. Fat arrows: light driven charge separation. Thin arrows: electron transfer reactions. Broken arrows: exchange of excited states between PSU's with open and closed centres. Barred rate constants refer to closed RC's.

$\left.\kappa_{-1}, \bar{\kappa}_{-1}\right)$ to the charge separated state $\left(\mathrm{Chl}-\mathrm{RC}^{+}\right) \mathrm{Phe}^{-} \mathrm{Q}_{A}^{(-)}$, or back to the ground state $\left(\kappa_{3}, \bar{\kappa}_{3}\right)$ via emission of fluorescence or other deactivation paths. If the reaction centre is "open", i.e. $\mathrm{Q}_{A}$ is oxidized, the $\mathrm{Phe}^{-}$charge is stabilized by transfer to $\mathrm{Q}_{A}^{-}\left(\kappa_{2}\right)$, and the electron hole at the $\mathrm{RC}$ is filled up $\left(\kappa_{4}\right)$ by donors fed by the water-splitting system. These steps are considered as irreversible in the context of prompt fluorescence (the small back reaction rates do, however, play an important part in delayed light emission, as reviewed by Jursinic, 1986).

$\mathrm{Q}_{A}$ can carry at most one electron (in contrast to $\mathrm{Q}_{B}$ which can carry two of them), so with $\mathrm{Q}_{A}$ reduced ("closed" reaction centre), the $\mathrm{Phe}^{-}$charge cannot be stabilized and recombines with the electron hole, whereby the excited state is $\left(\bar{\kappa}_{-1}\right)$ or is not $\left(\bar{\kappa}_{2}\right)$ repopulated [it is still controversial which of these (or both?) possibilities is actually realized; see Leibl et al., 1989].

The reaction scheme also includes the exchange of excited states between PSU's with open and closed centres $\left(\kappa_{5}, \bar{\kappa}_{5}\right.$; migration among open or among closed centres need not be considered because it induces no net flux). The existence of such an exchange has been a long-standing assumption though novel experiments have raised some doubt (see Joliot and Joliot, 1964; Baker and Webber, 1987; Black et al., 1986; and Krause and Weis, 1991, for recent reviews).

The model as proposed here assumes that energy transfer takes place at a certain rate between a PSU and all of its neighbours. This corresponds to a "lake model" (Baker and Webber, 1987) (if $\kappa_{5}, \bar{\kappa}_{5}>\kappa_{1}, \bar{\kappa}_{1}, \kappa_{3}, \bar{\kappa}_{3}$ ), to a model 
of isolated "separate pack" units (if $\kappa_{5}, \bar{\kappa}_{5}=0$ ), or else to a model of distinct but communicating units as proposed, e.g. in (Strasser, 1978). In the latter case, it is also a good approximation to a situation with a limited number of interacting units, as long as this number is not too small.

Our aim is to bring together both reaction schemes, which will be simplified by considering the fast sequence first. Its corresponding ODE system reads:

(Chl-RC)PheQ ${ }_{A}: \quad \dot{x}_{1}=\kappa_{5} \bar{x}_{1} x_{2}-\bar{\kappa}_{5} x_{1} \bar{x}_{2}-k_{L} x_{1}+\kappa_{3} x_{2}$

$(\mathrm{Chl}-\mathrm{RC})^{*} \mathrm{PheQ}_{A}: \quad \dot{x}_{2}=-\left(\kappa_{1}+\kappa_{3}+\kappa_{5} \bar{x}_{1}\right) x_{2}+\kappa_{-1} x_{3}+\bar{\kappa}_{5} x_{1} \bar{x}_{2}+k_{L} x_{1}$

$\left(\mathrm{Chl}^{-\mathrm{RC}^{+}}\right) \mathrm{Phe}^{-} \mathrm{Q}_{A}: \dot{x}_{3}=\kappa_{1} x_{2}-\left(\kappa_{-1}+\kappa_{2}\right) x_{3}$

$\left(\mathrm{Chl}^{-\mathrm{RC}^{+}}\right) \mathrm{PheQ}_{A}^{-}: \quad \dot{x}_{4}=\kappa_{2} x_{3}-\kappa_{4} x_{4}$

(Chl-RC)PheQ ${ }_{A}^{-}: \quad \dot{\bar{x}}_{1}=-\kappa_{5} \bar{x}_{1} x_{2}+\bar{\kappa}_{5} x_{1} \bar{x}_{2}-\bar{k}_{L} \bar{x}_{1}+\bar{\kappa}_{3} \bar{x}_{2}+\kappa_{4} x_{4}$

$(\mathrm{Chl}-\mathrm{RC})^{*} \mathrm{PheQ}_{A}^{-}: \quad \dot{\bar{x}}_{2}=-\left(\bar{\kappa}_{-1}+\bar{\kappa}_{3}+\bar{\kappa}_{5} x_{1}\right) \bar{x}_{2}+\bar{\kappa}_{1} \bar{x}_{3}+\kappa_{5} \bar{x}_{1} x_{2}+\bar{k}_{L} \bar{x}_{1}$

$\left(\mathrm{Chl}_{-\mathrm{RC}^{+}}\right) \mathrm{Phe}^{-} \mathrm{Q}_{A}^{-}: \dot{\bar{x}}_{3}=\bar{\kappa}_{1} \bar{x}_{2}-\left(\bar{\kappa}_{-1}+\bar{\kappa}_{2}\right) \bar{x}_{3}$.

With the $\kappa$ rate constants several orders of magnitude larger than the $k$ 's (the slowest $\kappa$ is $\kappa_{4}\left[\geqslant 4 \mu \mathrm{sec}^{-1}\right.$ as long as the turnover rate does not exhaust the electron donors (Schreiber and Neubauer, 1987) whereas the fastest $k$ is $k_{1} \approx 3.5 \mathrm{msec}^{-1}$ (Crofts and Wraight, 1983; Renger and Schulze, 1985)] and negligible concentrations of the intermediary states $x_{2}, \bar{x}_{2}, x_{3}, \bar{x}_{3}$ and $x_{4}$ to be expected, the time scales are safely enough separated for the intermediary states to be in quasi steady state with $x_{1}, \bar{x}_{1}$ on the time scale of measurement (milliseconds). This yields:

$$
\begin{aligned}
& x_{2}=x_{1} \frac{k_{L} \bar{\gamma}+\bar{\kappa}_{5}\left(k_{L} x_{1}+\bar{k}_{L} \bar{x}_{1}\right)}{\gamma \bar{\gamma}+\bar{\kappa}_{5} \gamma x_{1}+\kappa_{5} \bar{\gamma} \bar{x}_{1}} \\
& \bar{x}_{2}=\bar{x}_{1} \frac{\bar{k}_{L} \gamma+\kappa_{5}\left(k_{L} x_{1}+\bar{k}_{L} \bar{x}_{1}\right)}{\gamma \bar{\gamma}+\bar{\kappa}_{5} \gamma x_{1}+\kappa_{5} \bar{\gamma} \bar{x}_{1}} \\
& x_{3}=\frac{\kappa_{1}}{\kappa_{-1}+\kappa_{2}} x_{2} \\
& \bar{x}_{3}=\frac{\bar{\kappa}_{1}}{\bar{\kappa}_{-1}+\bar{\kappa}_{2}} \bar{x}_{2}
\end{aligned}
$$




$$
x_{4}=\frac{\kappa_{2}}{\kappa_{4}} x_{3}
$$

with

$$
\gamma=\kappa_{1}+\kappa_{3}-\frac{\kappa_{1} \kappa_{-1}}{\kappa_{-1}+\kappa_{2}}, \quad \bar{\gamma}=\bar{\kappa}_{1}+\bar{\kappa}_{3}-\frac{\bar{\kappa}_{1} \bar{\kappa}_{-1}}{\bar{\kappa}_{-1}+\bar{\kappa}_{2}}
$$

$(\gamma, \bar{\gamma}$ can be interpreted as the quasi steady-state de-excitation rate constants of excited states in PSU's with open or closed reaction centres, respectively).

With the additional assumption that $k_{L}=\bar{k}_{L}$, the expressions for $x_{2}$ and $\bar{x}_{2}$ simplify to:

$$
\begin{aligned}
& x_{2}=x_{1} \frac{k_{L}\left(\bar{\gamma}+\bar{\kappa}_{5}\right)}{\gamma \bar{\gamma}+\bar{\kappa}_{5} \gamma x_{1}+\kappa_{5} \bar{\gamma} \bar{x}_{1}} \\
& \bar{x}_{2}=\bar{x}_{1} \frac{k_{L}\left(\gamma+\kappa_{5}\right)}{\gamma \bar{\gamma}+\bar{\kappa}_{5} \gamma x_{1}+\kappa_{5} \bar{\gamma} \bar{x}_{1}}
\end{aligned}
$$

where we have used the normalization $x_{1}+\bar{x}_{1} \simeq 1$ (neglecting the concentrations of the intermediary states).

We have thus determined the quasi steady-state concentrations of the excited and charge separated intermediary states as functions of the fractions of open and closed reaction centres. This is what we need to find the rate function of the light step, $r$, and the fluorescence of the whole system.

The rate of $\mathrm{Q}_{A}$ reduction is $\kappa_{4} x_{4}$, so:

$$
r\left(x_{1}\right)=\frac{\kappa_{2} x_{3}}{x_{1}}=\left(\bar{\gamma}+\bar{\kappa}_{5}\right) \frac{\kappa_{1} \kappa_{2}}{\kappa_{-1}+\kappa_{2}} \cdot \frac{k_{L}}{\gamma \bar{\gamma}+\bar{\kappa}_{5} \gamma x_{1}+\kappa_{5} \bar{\gamma}\left(1-x_{1}\right)} .
$$

The fluorescence is a flux of photons resulting from the deactivation (via $\kappa_{3}, \vec{\kappa}_{3}$ ) of excited states:

$$
\begin{aligned}
F\left(x_{1}\right) & =S\left(\kappa_{3} q x_{2}+\bar{\kappa}_{3} \bar{q} \bar{x}_{2}\right) \\
& =\frac{S k_{L}}{\gamma \bar{\gamma}+\bar{\kappa}_{5} \gamma x_{1}+\kappa_{5} \bar{\gamma}\left(1-x_{1}\right)}\left(q \kappa_{3}\left(\bar{\gamma}+\bar{\kappa}_{5}\right) x_{1}+\bar{q} \bar{\kappa}_{3}\left(\gamma+\kappa_{5}\right) \bar{x}_{1}\right)
\end{aligned}
$$

where $S$ is a scaling factor due to the measuring apparatus, and $q$ and $\bar{q}$ are the probabilities that the excitation energy is emitted as fluorescence in the process of deactivation.

We additionally assume $\bar{\kappa}_{3}=\kappa_{3}$ (found experimentally in Leibl et al., 1989) and $\bar{\kappa}_{5}=\kappa_{5}, q=\bar{q}$ (which can hardly be conceived not to hold) and rescale the parameters: 


$$
\begin{aligned}
\tilde{S} & =S q \kappa_{3} \frac{\kappa_{-1}+\kappa_{2}}{\kappa_{1} \kappa_{2}} \cdot \frac{\gamma+\kappa_{5}}{\bar{\gamma}+\kappa_{5}} \\
\tilde{k}_{L} & =k_{L} \frac{\kappa_{1} \kappa_{2}}{\kappa_{-1}+\kappa_{2}} \cdot \frac{\bar{\gamma}+\kappa_{5}}{\left(\gamma+\kappa_{5}\right)^{2}} \\
\alpha & =\frac{\gamma-\bar{\gamma}}{\gamma+\kappa_{5}} \\
\beta & =\frac{\kappa_{5}}{\gamma+\kappa_{5}}
\end{aligned}
$$

where $\alpha$ can be interpreted as the relative difference between open and closed PSU's concerning the deactivation rate of excited states, and $\beta$ is the relative exciton exchange rate. This yields the much simpler expressions:

$$
\begin{aligned}
r\left(x_{1}\right) & =\frac{\tilde{k_{L}}}{1-\alpha-\beta+\alpha \beta x_{1}} \\
F\left(x_{1}\right) & =\tilde{S} \tilde{k}_{L} \frac{1-\alpha x_{1}}{1-\alpha-\beta+\alpha \beta x_{1}} \\
& =\tilde{S} \cdot\left(1-\alpha x_{1}\right) \cdot r\left(x_{1}\right) .
\end{aligned}
$$

The relationships (22) and (23) were already found by Strasser (1978) from a phenomenological model. Our derivation from the fast reaction scheme, however, enhances understanding by relating the parameters to the fast rate constants.

We are now ready to write down the complete core model for the fluorescence rise by translating Fig. 2 into its corresponding ODE system via mass action kinetics, and introducing (22) and (23):

$$
\begin{aligned}
& Q_{A} Q_{B}: \quad \dot{y}_{1}=-\left(r\left(x_{1}(t)\right)+k_{3}\left(C-y_{6}\right)\right) y_{1}+k_{-3} y_{5} y_{6} \\
& Q_{A}^{-} Q_{B}: \quad \dot{y}_{2}=r\left(x_{1}(t)\right) y_{1}-\left(k_{1}+k_{4}\left(C-y_{6}\right)\right) y_{2}+k_{-1} y_{3}+k_{-4} y_{6}\left(1-\sum_{i=1}^{5} y_{i}\right) \\
& Q_{A} Q_{B}^{-}: \dot{y}_{3}=k_{1} y_{2}-\left(r\left(x_{1}(t)\right)+k_{-1}\right) y_{3} \\
& Q_{A}^{-} Q_{B}^{-}: \dot{y}_{4}=r\left(x_{1}(t)\right) y_{3}-k_{2} y_{4}+k_{-2} y_{5}
\end{aligned}
$$




$$
\begin{aligned}
Q_{A} Q_{B}^{2-}: \dot{y}_{5}= & k_{3} y_{1}\left(C-y_{6}\right)+k_{2} y_{4}-\left(r\left(x_{1}(t)\right)+k_{-2}+k_{-3} y_{6}\right) y_{5} \\
\mathrm{PQ}_{\mathrm{ox}}: \quad \dot{y}_{6}= & -k_{-3} y_{5} y_{6}-k_{-4}\left(1-\sum_{i=1}^{5} y_{i}\right) y_{6}+k_{3} y_{1}\left(C-y_{6}\right) \\
& +k_{4} y_{2}\left(C-y_{6}\right)+k_{5}\left(C-y_{6}\right) .
\end{aligned}
$$

Here, we have used the normalizations:

$$
\left[Q_{A} Q_{B}\right]+\left[Q_{A}^{-} Q_{B}\right]+\left[Q_{A} Q_{B}^{-}\right]+\left[Q_{A}^{-} Q_{B}^{-}\right]+\left[Q_{A} Q_{B}^{2-}\right]+\left[Q_{A}^{-} Q_{B}^{2-}\right]=1
$$

and

$$
\left[\mathrm{PQ}_{\mathrm{ox}}\right]+\left[\mathrm{PQ}_{\mathrm{red}}\right]=C \text {. }
$$

The light reaction rate function has the form:

$$
r\left(x_{1}(t)\right)=\frac{\tilde{k}_{L}}{\left.1-\alpha-\beta+\alpha \beta x_{1}(t)\right)},
$$

where the fraction of open reaction centres is:

$$
x_{1}(t)=y_{1}(t)+y_{3}(t)+y_{5}(t),
$$

and, finally, the fluorescence reads:

$$
F\left(x_{1}(t)\right)=\tilde{S} \cdot\left(1-\alpha x_{1}\right) \cdot r\left(x_{1}\right) \text {. }
$$

This model is formally similar to that of Renger and Schulze (1985) to which it also owes the slow reaction scheme. In the Renger model, however, the fast reaction scheme is not considered explicitly, which gives rise to a severe inconsistency: Energy transfer between neighbouring units is taken into account as far as fluorescence is considered (which implies $x_{2}$ to depend on $x_{1}$ ), but $r$ is assumed to be constant with time [which implies $x_{2}$ to be independent of $x_{1}$, cf. (16)].

\section{Fitting the Model to Experimental Data.}

Experimental data. We deliberately chose intact leaf discs as our experimental objects. We are well aware of the fact that tackling the complex in vivo system should, in principle, be preceded by treating parts of the system separately. The standard simplification in this case is achieved by DCMU inhibition. However, DCMU in concentrations high enough to completely suppress $\mathrm{PQ}$ reduction has side-effects not yet fully understood, like, for example, lifting of the I level (see, for example, Neubauer and Schreiber, 1987). Such effects must be suspected to introduce more complications than simplifications into a quantitative comparison of models and data. 
The fluorescence rise curves were measured with standard methods as described in (Baake, 1989) and depicted in Fig. 4. Tobacco leaf discs were dark adapted and then reilluminated with the red (wavelength $633 \mathrm{~nm}$ ) light of a helium-neon laser at various intensities $\left(20,28\right.$ and $\left.40 \mathrm{~W} / \mathrm{m}^{2}\right)$; simultaneously the (unmodulated) fluorescence (wavelength $685 \mathrm{~nm}$ ) was recorded on line

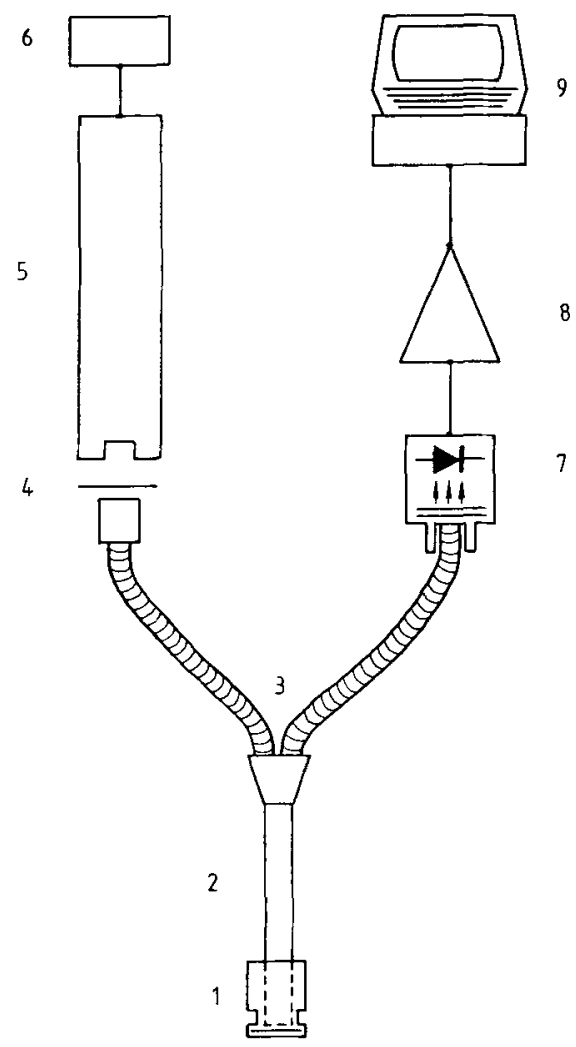

Figure 4. Experimental setup for measuring the fast fluorescence rise. 1 cuvette with leaf disc, 2, 3 fibre optics, 4 shutter, 5 HeNe laser, 6 power supply, 7 fluorescence detector with filter combination and photodiode, 8 amplifier, 9 computer.

from the upper leaf surface with a resolution of $1 \mathrm{msec}$. The measurement error was tested to be normally distributed with expectation 0 and constant variance $\sigma^{2}$.

A priori assumptions on parameters and initial values. The core model formally possesses the following degrees of freedom:

- the initial values $y_{1}(0), \ldots, y_{6}(0)$;

- the (scaled) rate of light absorption, $\tilde{k}_{L}$; 
- the rate constants of the (slow) redox reactions, $k_{1}, k_{-1}, k_{2}, k_{-2}, k_{3}, k_{-3}, k_{4}$, $k_{-4}$, and $k_{5}$;

- the joint parameters from the fast subsystem, $\alpha$ and $\beta$;

- the size of the PQ pool;

- and the scaling factor $\tilde{S}$.

Not all of them can be determined from curves such as in Fig. 1, but this is fortunately unnecessary as there is conclusive evidence concerning at least some of them. As discussed by Crofts and Wraight (1983), it is reasonable to assume $k_{3}=k_{-3}=k_{4}=k_{-4}$ (as is also assumed by Renger and Schulze, 1985). Furthermore, there is agreement on $k_{1} \simeq 3500 \mathrm{sec}^{-1}, k_{-1} \simeq 175 \mathrm{sec}^{-1}$ and $k_{2} \simeq 1750 \mathrm{sec}^{-1}$, but not on the value of $k_{-2}$. There is reasoning for $k_{-2}=1750 \mathrm{sec}^{-1}$ as well as for $k_{-2}=35 \mathrm{sec}^{-1}$ (Crofts and Wraight, 1983; Renger and Schulze, 1985). Anticipating that $k_{-2}$ cannot be determined from the data unambiguously, we are going to work with both values as fixed parameters for comparison.

Additional constraints are also reasonable for the initial values. In the dark adapted state, PQ is oxidized (Duysens, 1986), and about every fourth acceptor complex is singly reduced (Sane and Rutherford, 1986). This yields two constraints on the initial values:

$$
\begin{array}{r}
y_{2}(0)+y_{3}(0)=\frac{1}{4} \\
y_{6}(0)=C .
\end{array}
$$

The remaining initial values are determined by the condition that the electron transport chain be in equilibrium in the dark adapted $\left(k_{L}=0\right)$ state, so:

$$
\begin{aligned}
& y_{1}(0)=1-\left(y_{2}(0)+y_{3}(0)\right)=0.75 \\
& y_{2}(0)=\frac{1}{4} \frac{k_{-1}}{k_{1}}=0.0125 \\
& y_{3}(0)=\frac{1}{4}\left(1-\frac{k_{-1}}{k_{1}}\right)=0.2375 \\
& y_{4}(0)=y_{5}(0)=0 \\
& y_{6}(0)=C .
\end{aligned}
$$

With these constraints, the number of free parameters is reduced to seven: $k_{3}$, $k_{5}, C, \alpha, \beta, \tilde{k}_{L}$ and $\tilde{S}$. 
The problem of parameter estimation. In a multiple experiment approach (see Schlöder, 1988, for methods that exploit this structure), the model is fitted simultaneously to the measured induction curves at $n_{1}=3$ different light intensities. The ODE system then consists of 18 components, $y_{i}^{l}, i=1, \ldots, 6$, $l=1,2,3$, with initial values as in (35)-(39), and may be written as:

$$
\dot{y}^{l}=f\left(t, y^{l}, p_{\mathrm{s}}, p_{\mathrm{e}}^{l}\right),
$$

where $f$ is the right hand side of (24)-(29) and depends on the vector $p_{s}$ of system parameters $\left(k_{3}, C, \alpha, \beta, S\right)$ and the vector $p_{\mathrm{e}}$ of experiment-specific (i.e. intensity dependent) parameters, $\tilde{k}_{L}$ and $k_{5}\left(k_{5}\right.$ is intensity dependent because it summarizes the reoxidation of PQ via light reaction I).

Objective function. For each induction curve, $n_{2}=96$ observations $F_{M}^{l}\left(t_{j}\right)$ at equidistant instants $\left.t_{j}=(j-1) \times 0.005 \mathrm{sec}, j=1, \ldots, n_{2}\right)$ are taken into account, and the joint least squares objective function reads:

$$
\begin{aligned}
\mathscr{R}(y, p) & =\sum_{l=1}^{n_{1}} \sum_{j=1}^{n_{2}} \frac{1}{\sigma^{2}}\left(F_{M}^{l}\left(t_{j}\right)-F\left(y^{l}\left(t_{j}\right), p_{\mathrm{s}}, p_{\mathrm{e}}^{l}\right)\right)^{2} \\
& =:\left\|r_{1}\left(y^{1}\left(t_{1}\right), \ldots, y^{1}\left(t_{n_{2}}\right), \ldots, y^{n_{1}}\left(t_{1}\right), \ldots, y^{n_{1}}\left(t_{n_{2}}\right), p\right)\right\|_{2}^{2}
\end{aligned}
$$

( $r_{1}$ is the vector of the $n_{1} n_{2}$ least squares conditions).

With independent and normally distributed residuals, the minimization of $\mathscr{R}$ yields a maximum likelihood estimation of the unknown parameters.

Constraints. As reasoned above, the initial values for the $y_{i}^{l}$ are fixed at their estimated values. This yields $n_{3}=18$ equality constraints written as a vector $r_{2}$ with $n_{3}$ components. The parameter estimation problem such posed then yields a highly nonlinear, constrained least squares problem which may be summarized as:

$$
\begin{array}{rl}
\left\|r_{1}\left(y^{1}\left(t_{1}\right), \ldots, y^{1}\left(t_{n_{2}}\right), \ldots, y^{n_{1}}\left(t_{n_{2}}\right), \ldots, y^{n_{1}}\left(t_{n_{2}}\right), p\right)\right\|_{2}^{2} & =\min _{y, p} \\
r_{2}\left(y^{1}\left(t_{1}\right), \ldots, y^{n_{1}}\left(t_{1}\right), p\right) & =0 \\
\dot{y}^{l}= & f\left(t, y^{l}, p_{\mathrm{s}}, p_{\mathrm{e}}^{l}\right), \\
l & l, \ldots, n_{1} .
\end{array}
$$

Properties of the parameter estimation problem. The special parameter estimation problem under discussion here possesses some characteristic features. Firstly, the ODE system is highly nonlinear (see not only the products 
of components but also the structure of the light reaction rate function!) and stiff. Secondly, the model consists of six ODE components per experiment, opposed by only one measured quantity, so the problem can be expected to be ill-conditioned from the beginning. Furthermore, the measured quantity is a nonlinear function of a sum of components and contains a scaling factor which does not enter into the differential equation but must be estimated, too.

Solution of the parameter estimation problem. The features mentioned are some of the typical difficulties that occur in parameter estimation problems in systems of nonlinear ODE. They are accounted for in the boundary value problem methods developed by Bock and coworkers $(1981,1987)$ and Schlöder (1988) for this class of problems.

The multiple shooting method PARFIT as the most versatile member of this class of methods is described in, for example, Bock (1981), and a comprehensive treatment can be found in Bock (1987). Its basic idea is to treat the ODE constraint-independently of its specific nature - not as an initial value problem but as a multi point boundary value problem. To this end, the measuring interval $\left[t_{1}, t_{n_{2}}\right]$ is covered by a suitable grid of multiple shooting nodes $\tau$, such that $\tau_{1}<\tau_{2}<\ldots<\tau_{m},\left[t_{1}, t_{n_{2}}\right] \subset\left[\tau_{1}, \tau_{m}\right]$, and $m-1$ initial value problems are considered:

$$
\begin{aligned}
\dot{y} & =f(t, y, p), t \in\left[\tau_{j}, \tau_{j+1}\right] \\
y\left(t_{j}\right) & =s_{j}
\end{aligned}
$$

where the additional variables $s_{j}$ represent the state variables at the nodes. By choosing adequate initial guesses for them, any knowledge about the process, especially that inherent in the measurements, can directly be used in the formulation of the problem. Equations (45) and (46) mean that the problem is integrated piecewise, starting at the $s_{j}$, which yields, with the initial guesses for $s_{j}$ and $p$, a discontinuous initial trajectory (see Fig. 5). The task then consists in determining the $p$ and $s_{j}$ such that the objective function is minimized, the constraints are fulfilled, and the final trajectory is made continuous (see Fig. 6). Note that, in every iteration step, neither the trajectory is required to be continuous, nor the constraints to be fulfilled.

The basic problem (42)-(44) is thus reformulated as a large constrained least squares problem in the augmented variable vector $\left(p^{T}, s_{1}^{T}, \ldots, s_{m}^{T}\right)^{T}$, where $R_{1}$ corresponds to $r_{1}$ in (42):

$$
\begin{aligned}
\left\|R_{1}\left(s_{1}, \ldots, s_{m}, p\right)\right\|_{2}^{2} & =\min \\
R_{2}\left(s_{1}, \ldots, s_{m}, p\right) & =0 \quad \text { or } \geqslant 0
\end{aligned}
$$




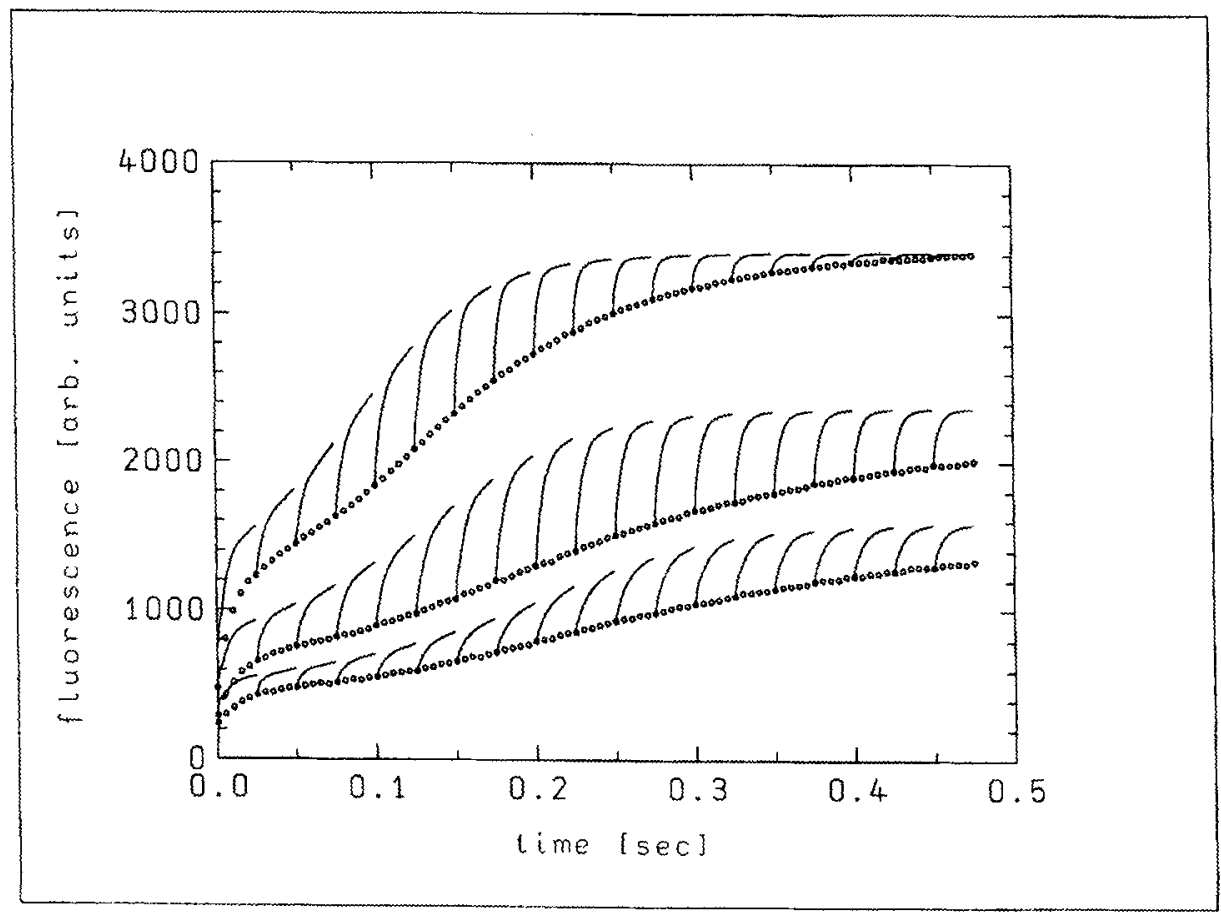

Figure 5. Initial curve of the multiple shooting algorithm. Dots: data, line: initial curve. Light intensities (from top to bottom): $40,28,20 \mathrm{~W} / \mathrm{m}^{2}$.

$$
y\left(\tau_{j+1}, s_{j}, p\right)-s_{j+1}=0, j=1, \ldots, m-1
$$

where the conditions (49) ensure the continuity of the final trajectory, and (48) summarizes all pointwise equality and possible inequality conditions on trajectory and parameters.

Thus a constrained nonlinear least squares problem of the general form:

$$
\begin{aligned}
\left\|u_{1}(x)\right\|_{2}^{2} & =\min \\
u_{2}(x) & =0 \quad \text { or } \geqslant 0
\end{aligned}
$$

is obtained, which is solved by a generalized Gauss-Newton-method as described and analysed in Bock (1987). Starting from an initial guess $x^{0}$, the vector of variables $x$ is iterated via:

$$
x^{k+1}=x^{k}+\lambda^{k} \Delta x^{k}
$$

where the $\lambda^{k} \in[0,1]$ are damping factors which are determined via so-called level functions to ensure global convergence (see Bock, 1987), and $\Delta x^{k}$ solves the linearized problem: 


$$
\begin{aligned}
\left\|u_{1}\left(x^{k}\right)+\frac{\partial u_{1}}{\partial x}\left(x^{k}\right) \Delta x^{k}\right\|_{2}^{2} & =\min \\
u_{2}\left(x^{k}\right)+\frac{\partial u_{2}}{\partial x}\left(x^{k}\right) \Delta x^{k} & =0 \quad \text { or } \geqslant 0 .
\end{aligned}
$$

In the region of local convergence $\left(\lambda^{k}=1\right)$, the algorithm converges linearly to a statistically stable solution.

The integrations required are carried out with a stiff integrator based on METAN by Bader and Deuflhard (1981), which also performs the differentiation via so-called internal numerical differentiation as introduced in Bock (1981). This is essentially a differentiation of the internally generated discretization scheme itself, which demands no derivations from the user, is fast and accurate. Note that, although the multiple shooting approach has much more variables than an initial value problem approach, the integration and linearization effort is essentially not increased, which even holds in the case of fixed initial values (Schlöder, 1988).

Numerical results. The initial parameter estimates (see Table 1) are chosen in accordance with Renger and Schulze (1985). We choose 20 equidistant

Table 1. Initial estimates for parameters. All rate constants are in $\mathrm{sec}^{-1}$

\begin{tabular}{rrrrrrrr}
\hline Light & $\tilde{k}_{L}$ & $k_{3}$ & $k_{5}$ & $C$ & $\beta$ & $\alpha$ & $\tilde{S}$ \\
\hline $40 \mathrm{~W} / \mathrm{m}^{2}$ & 150.0 & & 1.0 & & & & \\
$28 \mathrm{~W} / \mathrm{m}^{2}$ & 105.0 & 30.0 & 1.0 & 10.0 & 0.5 & 0.4 & 2.4 \\
$20 \mathrm{~W} / \mathrm{m}^{2}$ & 75.0 & & 1.0 & & & & \\
\hline
\end{tabular}

multiple shooting nodes $\tau_{j}, \tau_{j}=(j-1) \times 0.025 \mathrm{sec}$. Apart from $\tau_{1}\left(=t_{1}\right)$, where the initial conditions are fixed anyway, estimates for the state variables are required at these nodes. As there is only one measured quantity available for every six ODE components, which is itself a function of a sum of three components, we only obtain a crude approximation via the inverse function:

$$
\begin{gathered}
\hat{x}_{1}^{l}\left(\tau_{j}\right)=\frac{F_{M}^{l}\left(\tau_{j}\right)(\beta-\alpha+1)+\tilde{k}_{L}^{l} \tilde{S}}{\alpha \beta F_{M}^{l}\left(\tau_{j}\right)+\alpha \tilde{k}_{L}^{l} \tilde{S}} \\
\hat{y}_{1}^{l}\left(\tau_{j}\right)=\hat{y}_{3}^{l}\left(\tau_{j}\right)=\hat{y}_{5}^{l}\left(\tau_{j}\right):=\frac{\hat{x}_{1}\left(\tau_{j}\right)}{3}, \quad l=1,2,3, \quad j=2, \ldots, 20 .
\end{gathered}
$$

For the alternative $k_{-2}=k_{2}$, we can additionally assume $\hat{y}_{4}^{l}\left(\tau_{j}\right):=\hat{y}_{5}^{l}\left(\tau_{j}\right)$; for the 
other components, we take the results of a test integration from the preceding interval:

$$
\hat{y}_{i}^{l}\left(\tau_{j+1}\right)=y_{i}^{l}\left(\tau_{j+1} ; s_{j}, p\right), \quad i=2, \quad i=6, \quad j=2, \ldots, 20, \quad l=1,2,3 .
$$

With these initial estimates for the parameters and components, we obtain initial trajectories for the fluorescence as shown in Fig. 5.

The result of the fitting procedure is shown in Fig. 6 and Table 2. The outcome for a second data set is very similar, which demonstrates the reproducibility of the measurement and the fitting procedure. With deviations from the data of only $0.9-2.4 \%$, the fits are remarkably good, and the parameter estimates are, on the whole, realistic, as detailed below.

C. In the literature, the stoichiometry of PQ molecules per PS II reaction centre is generally taken to be $7-10$ (Crofts and Wraight, 1983) and is overestimated in the fits ( $C=12$ and $C=16$, respectively). This might result from neglecting of PS I and its acceptor pool, or from neglecting oxidized PQ as a fluorescence quencher (see below).

$k_{3}$. The product $k_{3} C$, which defines the half life for the reoxidation of $\mathrm{Q}_{B}$ by the PQ pool via $\tau_{1 / 2}=\log 2 / k_{3} C$, is astonishingly constant for both choices of $k_{-2}: \tau_{1 / 2} \simeq 3.3 \mathrm{msec}$, just slighly longer than the $2-3 \mathrm{msec}$ given by Haehnel (1984).

$\alpha, \beta$. It is interesting to note the connection between $\alpha, \beta$ and $k_{-2}$. In any case, $\alpha+\beta \simeq 0.9$, but $\beta$ nearly vanishes at $k_{-2}=35$ but is $\simeq 0.4$ at $k_{-2}=$ $1750 \mathrm{sec}^{-1}$. In the Renger model (1985), no satisfactory fit is achieved at all with the latter choice, which might be attributed to the fact that, in this model, the energy transfer is not modelled consistently.

Anyway, there is now considerable disagreement as to whether or not energy transfer between neighbouring PSU's takes place at all (see above).

$k_{-2}$. The nearly equally good fits for both choices suggest (and numerical experiments corroborate) that this parameter cannot be determined from these data independently of the other parameters. On the other hand, its influence on the other parameters demonstrate that it would be worthwhile to design different experiments to find out-perhaps with flash series and microsecond resolution.

4. Model Refinements. The curve fittings look excellent at first sight, but at a closer look, deviations are evident. Though small, they must be taken seriously, as they occur systematically (most pronounced at the lowest intensity) in all 

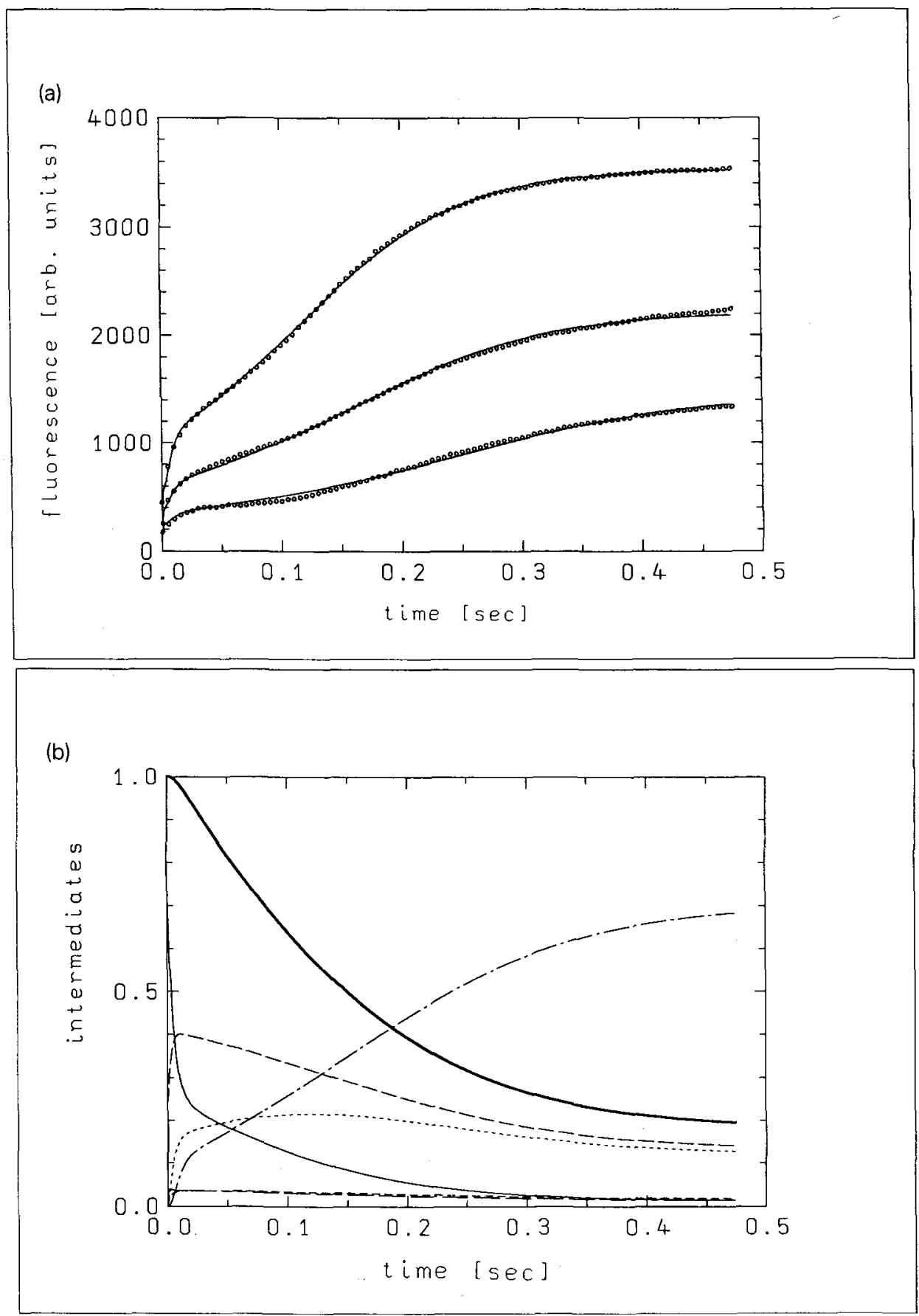

Figure 6. Fitted curve for dataset $2, k_{-2}=35 \mathrm{sec}^{-1}$. (a) Fluorescence. Intensities as in Fig. 5. (b) Concentrations of the species at $28 \mathrm{~W} / \mathrm{m}^{2}$. $\mathrm{Q}_{A} \mathrm{Q}_{B} ;-\overline{\mathrm{Q}_{A}^{-}} \mathrm{Q}_{B}^{2-}$ $\mathrm{Q}_{A}^{-} \mathrm{Q}_{B} ;-\cdots \mathrm{Q}_{A} \mathrm{Q}_{B}^{-} ; \cdots-\mathrm{Q}_{A}^{-} \mathrm{Q}_{B}^{-} ; \ldots \ldots$ 
Table 2. Parameter estimates, confidence intervals, and deviation from the data. All rate constants are in $\sec ^{-1}$. (a) Dataset $1, k_{-2}=1750$, (b) dataset $2, k_{-2}=1750$, (c) dataset 1 , (a)

$$
k_{-2}=35 \text {, (d) dataset } 2, k_{-2}=35
$$

\begin{tabular}{lcrrrrrrrr}
\hline & Light & $\tilde{k}_{L}$ & $k_{3}$ & $k_{5}$ & $C$ & $\beta$ & $\alpha$ & $\tilde{S}$ & Dev. \\
\hline \multirow{4}{*}{ Estimates } & $40 \mathrm{~W} / \mathrm{m}^{2}$ & 137.0 & & 1.94 & & & & & \\
& $28 \mathrm{~W} / \mathrm{m}^{2}$ & 97.0 & 12.9 & 2.30 & 16.8 & 0.462 & 0.486 & 1.61 & $1.4 \%$ \\
& $20 \mathrm{~W} / \mathrm{m}^{2}$ & 78.0 & & 2.37 & & & & & \\
$95 \%$ c.i. & $40 \mathrm{~W} / \mathrm{m}^{2}$ & 38.0 & & 1.10 & & & & & \\
& $28 \mathrm{~W} / \mathrm{m}^{2}$ & 25.0 & 1.6 & 0.78 & 4.2 & 0.16 & 0.14 & 0.37 & \\
& $20 \mathrm{~W} / \mathrm{m}^{2}$ & 20.0 & & 0.54 & & & & & \\
\hline
\end{tabular}

(b)

\begin{tabular}{lcrrrrrrrr}
\hline & Light & $\tilde{k}_{L}$ & $k_{3}$ & $k_{5}$ & $C$ & $\beta$ & $\alpha$ & $\tilde{S}$ & Dev. \\
\hline \multirow{4}{*}{ Estimates } & $40 \mathrm{~W} / \mathrm{m}^{2}$ & 181.0 & & 0.826 & & & & & \\
& $28 \mathrm{~W} / \mathrm{m}^{2}$ & 134.0 & 14.6 & 1.87 & 15.7 & 0.345 & 0.597 & 1.2 & $2.4 \%$ \\
& $20 \mathrm{~W} / \mathrm{m}^{2}$ & 96.2 & & 1.76 & & & & & \\
$95 \%$ c.i. & $40 \mathrm{~W} / \mathrm{m}^{2}$ & 27.0 & & 1.1 & & & & & \\
& $28 \mathrm{~W} / \mathrm{m}^{2}$ & 21.0 & 1.8 & 0.71 & 5.0 & 0.17 & 0.15 & 0.52 & \\
& $20 \mathrm{~W} / \mathrm{m}^{2}$ & 18.0 & & 0.48 & & & & & \\
\hline
\end{tabular}

(c)

\begin{tabular}{lcrrrrrrrr}
\hline & Light & $\tilde{k}_{L}$ & $k_{3}$ & $k_{5}$ & $C$ & $\beta$ & $\alpha$ & $\tilde{S}$ & Dev. \\
\hline \multirow{4}{*}{ Estimates } & $40 \mathrm{~W} / \mathrm{m}^{2}$ & 175.0 & & 2.65 & & & & & \\
& $28 \mathrm{~W} / \mathrm{m}^{2}$ & 122.0 & 16.7 & 2.63 & 12.3 & 0.085 & 0.831 & 2.19 & $2.0 \%$ \\
& $20 \mathrm{~W} / \mathrm{m}^{2}$ & 97.0 & & 2.47 & & & & & \\
$95 \%$ c.i. & $40 \mathrm{~W} / \mathrm{m}^{2}$ & 27.0 & & 1.10 & & & & & \\
& $28 \mathrm{~W} / \mathrm{m}^{2}$ & 21.0 & 4.2 & 0.71 & 3.8 & 0.075 & 0.070 & 0.54 & \\
& $20 \mathrm{~W} / \mathrm{m}^{2}$ & 18.0 & & 0.48 & & & & & \\
\hline
\end{tabular}

(d)

\begin{tabular}{cccccccccc}
\hline & Light & $\tilde{k}_{L}$ & $k_{3}$ & $k_{5}$ & $C$ & $\beta$ & $\alpha$ & $\tilde{S}$ & Dev. \\
\hline \multirow{3}{*}{ Estimates } & $40 \mathrm{~W} / \mathrm{m}^{2}$ & 199.0 & & 1.31 & & & & & \\
& $28 \mathrm{~W} / \mathrm{m}^{2}$ & 146.0 & 18.1 & 2.06 & 11.6 & 0.0564 & 0.862 & 1.64 & $0.9 \%$ \\
& $20 \mathrm{~W} / \mathrm{m}^{2}$ & 103.0 & & 1.75 & & & & & \\
$95 \%$ c.i. & $40 \mathrm{~W} / \mathrm{m}^{2}$ & 40.0 & & 1.50 & & & & & \\
& $28 \mathrm{~W} / \mathrm{m}^{2}$ & 31.0 & 4.0 & 1.10 & 3.8 & 0.063 & 0.063 & 0.50 & \\
\hline
\end{tabular}


cases examined, and the data are too precise for measurement error to be blamed.

We conclude that the core model yields a good approximation of the induction kinetics but fails to faithfully reproduce the ID dip where it is most pronounced, that is, at lower intensities. We now proceed to model refinements accounting for the two most prominent hypotheses attempting to explain the dip, that is, the PS I and the non-B-type hypothesis, asking whether they are able to remedy the situation.

The PS I hypothesis. The oldest hypothesis (and the one still most popular) ascribes the ID phase to the reoxidation of PS II acceptors by PS I (actually, Kautsky and coworkers concluded in 1960 from the OIDP kinetics that two spatially separated light reactions must exist; Duysens, 1986). Several lines of experimental evidence (for review, see Govindjee and Satoh, 1986) support the view that PS I, at the onset of illumination, drains many electrons from the PQ pool, until its own electron acceptors (the pool of ferredoxin) are saturated; this drainage is believed to lower the fluroescence via the redox state of $Q_{B}$ and $Q_{A}$ and thus produce the "dip" effect.

This mechanism is incorporated into the model as sketched in Fig. 7. As

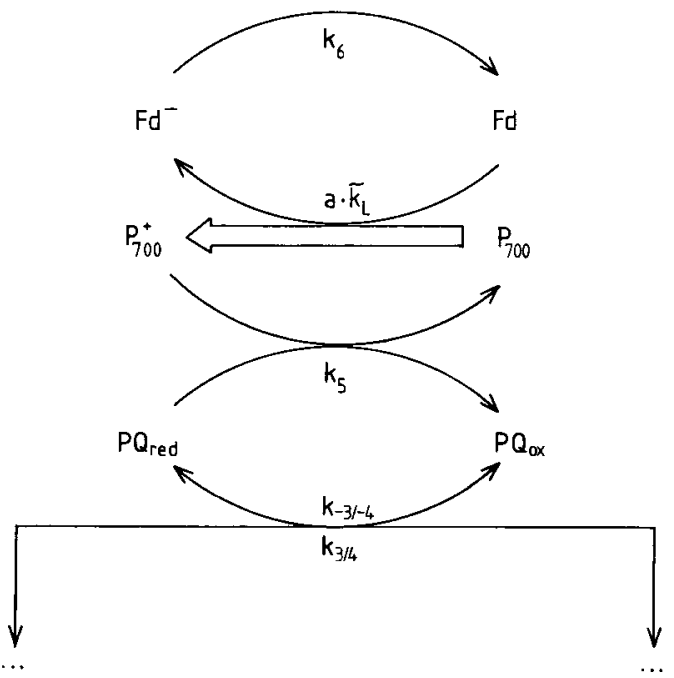

Figure 7. Reoxidation of plastoquinone via photosystem I. PQ: plastoquinone. $\mathrm{P}_{700}$ : photosystem I reaction centre. Fd: ferredoxin.

there is no energy transfer in PS I (cf. Baker and Webber, 1987), light driven charge separation is simply described by a rate constant $\left(a \tilde{k}_{L}\right.$, where $a$ is a factor relating the absorption cross section of PS I to that of PS II). It leads to oxidation of the reaction centre $\left(\mathrm{P}_{700}\right)$ and reduction of one molecule of 
ferredoxin $(\mathrm{Fd})$. The latter one is reoxidized via several reactions, represented here by a single quasi linear one $\left(k_{6}\right)$. The $\mathrm{P}_{700}^{+}$electron hole is filled up by electrons from the PQ pool via a sequence of reactions (see, for example, Baker and Webber, 1987) approximated here by a single second order reaction $\left(k_{5}\right)$.

The ODE system then reads:

$$
\begin{aligned}
\dot{y}_{1}= & \\
\vdots & {[\text { as in }(24)-(28)] } \\
\dot{y}_{5}= & \\
\mathrm{PQ}_{\mathrm{ox}}: \quad \dot{y}_{6}= & -k_{-3} y_{5} y_{6}-k_{-4}\left(1-\sum_{i=1}^{5} y_{i}\right) y_{6}+k_{3} y_{1}\left(C-y_{6}\right)+ \\
& +k_{4} y_{2}\left(C-y_{6}\right)+\frac{1}{2} k_{5}\left(C-y_{6}\right) y_{7} \\
\mathrm{P}_{700}^{+}: \quad \dot{y}_{7}= & a \tilde{k_{L}}\left(1-y_{7}\right) y_{8}-k_{5}\left(C-y_{6}\right) y_{7} \\
\mathrm{Fd}^{-}: \quad \dot{y}_{8}= & a \tilde{k}_{L}\left(1-y_{7}\right) y_{8}-k_{6}\left(D-y_{8}\right) \\
& y_{7}(0)=y_{8}(0)=0
\end{aligned}
$$

where the normalization $[\mathrm{Fd}]+\left[\mathrm{Fd}^{-}\right]=D(D$ size of the ferredoxin pool $)$ has been used and a 1:1 stoichiometry between PS I and PS II reaction centres has been assumed.

$D, k_{6}$, and $a$ have been introduced as additional parameters. On the other hand, $k_{5}$ is now a system parameter, so the actual number of free parameters is only increased by one.

We choose initial guesses plausible from the literature (Böhme, 1978; Govindjee and Satoh, 1986): $D=5, k_{6}=2, a=0.5$. The parameter estimation algorithm yields a fit which is somewhat worse than that of the core model, and parameter values $k_{3}=200, C=0.3$ and $k_{5} \simeq 4000 \mathrm{sec}^{-1}$. So the procedure tends to eliminate the PQ pool from the system by decreasing the pool size and increasing the corresponding exchange rates, thus attempting to make the communication between PS I and PS II as direct as possible, which cannot be considered to be realistic.

We conclude that the PS I hypothesis, as it stands, is not the whole story to the ID dip.

The non-B-type hypothesis. Quite a different view of the OIDP kinetics dates back to investigations by Forbush and Kok (1968) (and was confirmed 
later on by Lavergne, 1982a,b) who observed that the intensity dependence of the I niveau was not consistent with its being produced solely by the interaction of PS II and PQ. From the current point of view (Briantais, 1986), this observation reflects the heterogeneity of PS II electron transport. There exist two subpopulations of PS II: the major one (about three-quarters of the whole PS II), the so called B-type centres, work according to the two electron gate mechanism which is covered by our core model. A minor part, however, the non-B-type centres, do not possess $\mathrm{Q}_{B}$ (hence their name) and thus are not connected to the PQ pool, and their electron transport does not seem to go beyond the primary stable acceptor. Moreover, their antenna systems are smaller than those of the B-type centres, and they do not communicate with each other. For reviews of PS II heterogeneity, see Black et al. (1986) and Baker and Webber (1987).

Under these circumstances, the fluorescence induction due to the non-B-type fraction should be a simple exponential increase, and the fluorescence from (32) must be modified via:

$$
\widetilde{F}(t)=F(t)+R \tilde{k}_{L}\left(1-\tilde{\alpha} e^{-\tilde{a} \tilde{k}_{L} t}\right)
$$

where $R$ is a scaling factor including the ratio of B-type to non-B-type centres, $\tilde{a}$ is a factor relating light intensity to the non-B acceptor reduction rate, and $\tilde{\alpha}$ plays the role of $\alpha$ for the B-type centres.

Parameters consistent with the literature (Baker and Webber, 1987; Black $e t$ al., 1986) are $R=6, \tilde{a}=0.3$ and $\tilde{\alpha}=0.8$. Using these as our initial guess, we obtain a slightly better fit than before but with a negative $R$. To fix things, the fitting procedure is repeated with $R$ kept at a small positive value $(R=0.0001)$, which yields a solution with $\tilde{a} \simeq 4$ and $\tilde{\alpha} \simeq-6000$ ! It goes without saying that solutions implying negative rate constants need not be considered further.

So this model variant does not seem to be a good candidate for improving the model either. From a qualitative point of view, it could have been argued from the beginning that an additional exponential increase will tend to smooth out the "dip" instead of enhancing it.

Let us mention shortly that several other effects discussed in the context of the fast fluorescence rise were tentatively incorporated into the core model but did not lead to a better description of the data either. For example, oxidized PQ is thought to act as a non-photochemical quencher of fluorescence (van Gorkom, 1986). When this mechanism is included (via a formalism similar to that in Kischkoweit et al., 1988), this yields a more realistic value for $C(C \simeq 10)$, but no visible improvement of the fitted kinetics. Also, we modelled the effects of increasing $\mathrm{pH}$ in the stroma by including the protonation pattern of the twoelectron-gate sequence (as far as this is resolved to date, see Govindjee and Eaton-Rye, 1986), but this, too, hardly affects the kinetics of the fluorescence rise. 
5. Conclusion. Our core model is a pure PS II model accounting for the fast steps of excitation energy transfer and primary charge separation as well as the slower steps of electron transport through the two-electron-gate. The model is fitted to measured induction curves with a reliable fitting procedure which bears the great advantage of allowing a rigorous comparison of models and data, and a funded discrimination of different models. We obtain remarkably good fits to measured induction curves at moderate light intensities, which has not been achieved by former models. Nevertheless, small but systematic deviations remain, which are more conspicuous at the lower intensities (and which would be much more pronounced in experimental situations where the "dip" not only shows up as a shoulder but as a transient decrease in fluorescence described, for example, by Schreiber et al., 1971). These deviations are taken as a touchstone to test the validity of the two most prominent hypotheses trying to explain the OIDP behaviour. We find that these hypotheses - in their current formulation - do not yield a better description of the data than does the core model.

It can only be speculated which aspects might be truely responsible for the observed behaviour. Perhaps PS I is not the most likely candidate, at least not in its classical role in linear electron transport. This is corroborated by the observation that the typical ID dip also occurs in the presence of DBMIB (McCauley et al., 1987), an inhibitor which blocks the reoxidation of PQ.

Further models will not be allowed to ignore the heterogeneity of PS II, which is getting exceedingly more complex the more results are collected (Baker and Webber, 1987). The actual function of the enigmatic non-B-type centres and their electron transport still remains to be elucidated.

The effect of decreasing luminal $\mathrm{pH}$, which can easily be conceived to produce some "dip" by slowing down PS II reoxidation in the course of the illumination, is probably ruled out because it should be more pronounced at higher intensities. Actually, limitations by the donor side have been demonstrated to play a role at extreme light intensities (Schreiber and Neubauer, 1987).

A candidate for further investigation might be the membrane potential associated with the primary charge separation, which has recently been found to influence the fluorescence yield by shifting the energy levels of the species in Fig. 3 with respect to each other, thus influencing the rate constants of the fast subsystem (Dau et al., 1992; Leibl et al., 1989).

Discomforting is the fact that there is still considerable disagreement concerning even the global features of photosynthetic electron transport. The possibility of cyclic electron transport around PS II proposed by Arnon and Tang (1988) and McCauley et al. (1987) remains a great challenge to the common picture of linear electron transport.

Anyway, the Gordian knot posed by Kautsky may be expected to occupy experimentalists and theoreticians for another while. 
E.B. wishes to thank R. J. Strasser for encouraging her to undertake this work, for supporting her in performing the experiments in his laboratory, and for helpful discussions. We are very grateful to $\mathrm{W}$. Alt for his permanent encouragement and his constructive ideas during the progress of the theoretical work, and to H. G. Bock for stimulating discussions on numerical methods for nonlinear parameter estimation problems. Last but not least, we wish to thank H. Dau, U. P. Hansen, and U. Schreiber for valuable discussions on the physiology of the light reaction.

Financially, this work was supported by the Deutsche Forschungsgemeinschaft.

\section{LITERATURE}

Arnon, D. I. and G. M.-S. Tang. 1988. Cytochrome b-559 and proton conductance in oxygenic photosynthesis. Proc. natn. Acad. Sci. U.S.A. 85, 9524-9528.

Bader, G. and P. Deufhard. 1981. A semi-implicit mid-point rule for stiff systems of $O D E, S F B$ 123. Technical Report 114, University of Heidelberg, 1981.

Baake, E. 1989. Differentialgleichungssystem zur Beschreibung der Fluoreszenzinduktion (OIDP-Kinetik) der Photosynthese. PhD Theses, University of Bonn (in German).

Baker, N. R. and A. N. Webber. 1987. Interactions between photosystems. Adv. Bot. Res. 13, 1-66.

Black, M. T., T. H. Brearley and P. Horton. 1986. Heterogeneity in chloroplast photosystem II. Photosynth. Res. 8, 193-207.

Böhme, H. 1978. Quantitative determination of ferredoxin, ferredoxin-NADP ${ }^{+}$-reductase and plastocyanin in spinach chloroplasts. Eur. J. Biochem. 83, 137-141.

Bock, H. G. 1981. Numerical treatment of inverse problems in chemical reaction kinetics. In Modelling of Chemical Reaction Systems, K. H. Ebert, P. Deuflhard and W. Jäger (Eds), pp. 102-125. Berlin: Springer.

Bock, H. G. 1987. Randwertproblemmethoden zur Parameteridentifizierung in Systemen nichtlinearer Differentialgleichungen. Bonner Mathematische Schriften, Vol. 183. E. Brieskorn et al. (Eds) (in German).

Briantais, J.-M. et al. 1986. Chlorophyll a fluorescence of higher plants: chloroplasts and leaves. In Light Emission by Plants and Bacteria, Govindjee, J. Amesz and D. C. Fork (Eds). New York: Academic Press.

Crofts, A. R. and C. A. Wraight. 1983. The electrochemical domain of photosynthesis. Biochim. Biophys. Acta 726, 149-185.

Dau, H., R. Windecker and U. P. Hansen. 1992. Effect of light-induced changes in thylakoid voltage on chlorophyll fluorescence of Aegopodium podagraria leaves. Biochim. Biophys. Acta, in press.

Duysens, L. M. N. 1986. Introduction to (bacterio)chlorophyll emission: A historical perspective. In Light Emission by Plants and Bacteria, Govindjee, J. Amesz and D. C. Fork (Eds). New York: Academic Press.

Forbush, B. and B. Kok. 1968. Reaction between primary and secondary electron acceptors of photosystem II of photosynthesis. Biochim. Biophys. Acta 162, 243-253.

van Gorkom, H. 1986. Fluorescence measurements in the study of photosystem II electron transport. In Light Emission by Plants and Bacteria, Govindjee, J. Amesz and D. C. Fork (Eds). New York: Academic Press.

Govindjee and J. J. Eaton-Rye. 1986. Electron transfer through photosystem II acceptors: Interaction with anions. Photosynth. Res. 10, 365-379.

Govindjee and K. Satoh. 1986. Fluorescence properties of chlorophyll b- and chlorophyll 
c-containing algae. In Light Emission by Plants and Bacteria, Govindjee, J. Amesz and D. C. Fork (Eds). New York: Academic Press.

Haehnel, W. 1984. Photosynthetic electron transport. Ann. Rev. Plant Physiol. 35, 659-693.

Joliot, P. and A. Joliot. 1964. Etudes cinétiques de la réaction photochimique liberant l'oxygène au cours de la photosynthèse. C. R. Acad. Sci. Paris 258, 4622-4625.

Jursinic, P. A. 1986. Delayed fluorescence: Current concepts and status. In Light Emission by Plants and Bacteria, Govindjee, J. Amesz and D. C. Fork (Eds). New York: Academic Press.

Kautsky, H. and A. Hirsch. 1931. Neue Versuche zur Kohlensäureassimilation. Naturwissenschaften 48, 964-981.

Kischkoweit, C., W. Leibl and H. W. Trissl. 1988. Theoretical and experimental study of trapping times and antenna organization in pea chloroplasts by means of the artificial fluorescence quencher m-dinitrobenzene. Biochim. Biophys. Acta 933, 276-287.

Krause, G. H. and E. Weis. 1991. Chlorophyll fluorescence and photosynthesis: The basics. Ann. Rev. Plant Physiol. Plant Mol. Biol. 42, 313-349.

Lavergne, L. 1982a. Two types of primary acceptors in chloroplast photosystem II. I. Different recombination properties. Photobiochem. Photobiophys. 3, 257-271.

Lavergne, L. 1982b. Two types of primary acceptors in chloroplast photosystem II. II. Reduction in two successive photoacts. Photobiochem. Photobiophys. 3, 273-285.

Leibl, W., J. Breton, J. Deprez and H. W. Trissl. 1989. Photoelectric study on the kinetics of trapping and charge stabilization in oriented PS II membranes. Photosynth. Res. 22, $257-275$.

McCauley, S. W., A. Melis, G. H. S. Tang and D. I. Arnon. 1987. Protonophores induce plastoquinol oxidation and quench chloroplast fluorescence: Evidence for a cyclic, protonconducting pathway in oxygenic photosynthesis. Proc. natn. Acad. Sci. U.S.A. 84, 84248428 .

Neubauer, C. and U. Schreiber. 1987. The polyphasic rise of chlorophyll fluorescence upon onset of strong continuous illumination: I. Saturation characteristics and partial control by the photosystem II acceptor side. Z. Naturforsch. 42c, 1246-1254.

Renger, G. and A. Schulze. 1985. Quantitative analysis of fluorescence induction curves in isolated spinach chloroplasts. Photobiochem. Photobiophys. 9, 79-87.

Renger, G. and U. Schreiber. 1986. Practical applications of fluorometric methods to algae and higher plant research. In Light Emission by Plants and Bacteria, Govindjee, A. Amesz and D. C. Fork (Eds). New York: Academic Press.

Sane, P. V. and A. W. Rutherford. 1986. Thermoluminescence from photosynthetic membranes. In Light Emission by Plants and Bacteria, Govindjee, J. Amesz and D. C. Fork (Eds). New York: Academic Press.

Schatz, G. H., H. Brock and A. R. Holzwarth. 1988. Kinetic and energetic model for the primary processes in photosystem II. Biophys. J. 54, 397-405.

Schlöder, J. P. 1988. Numerische Methoden zur Behandlung hochdimensionaler Aufgaben der Parameteridentifizierung. Bonner Mathematische Schriften, Vol. 187, E. Brieskorn et al. (Eds) (in German).

Schreiber, U., R. Bauer and U. F. Franck. 1971. Chlorophyll fluorescence induction in green plants at oxygen deficiency. Proceedings of the Ind International Congress on Photosynthesis, Stresa 1971, pp. 169-179.

Schreiber, U. and C. Neubauer. 1987. The polyphasic rise of chlorophyll fluorescence upon onset of strong continuous illumination: II. Partial control by the photosystem II donor side and possible ways of interpretation. Z. Naturforsch. 42c, 1255-1264.

Strasser, R. J. 1978. The grouping model of plant photosynthesis. In Chloroplast Development, G. Akoyunoglou et al. (Eds). North Holland: Biomedical Press, Elsevier. 